\title{
Measuring children's physical activity
}

\section{Compliance using skin-taped accelerometers}

Schneller, Mikkel Bo; Bentsen, Peter; Nielsen, Glen; Brønd, Jan Christian; Ried-Larsen, Mathias; Mygind, Erik; Schipperijn, Jasper

Published in:

Medicine and Science in Sports and Exercise

DOI:

10.1249/MSS.0000000000001222

Publication date:

2017

Document version

Publisher's PDF, also known as Version of record

Document license:

CC BY

Citation for published version (APA):

Schneller, M. B., Bentsen, P., Nielsen, G., Brønd, J. C., Ried-Larsen, M., Mygind, E., \& Schipperijn, J. (2017).

Measuring children's physical activity: Compliance using skin-taped accelerometers. Medicine and Science in Sports and Exercise, 49(6), 1261-1269. https://doi.org/10.1249/MSS.0000000000001222 


\title{
Measuring Children's Physical Activity: Compliance Using Skin-Taped Accelerometers
}

\author{
MIKKEL BO SCHNELLER ${ }^{1,2}$, PETER BENTSEN ${ }^{1}$, GLEN NIELSEN ${ }^{3}$, JAN CHRISTIAN BRØND ${ }^{4}$, \\ MATHIAS RIED-LARSEN ${ }^{5,6}$, ERIK MYGIND ${ }^{7}$, and JASPER SCHIPPERIJN ${ }^{2}$
}

${ }^{1}$ Health Promotion Research, Steno Diabetes Center Copenhagen, Gentofte, DENMARK; ${ }^{2}$ Active Living, Department of Sports Science and Clinical Biomechanics, University of Southern Denmark, Odense, DENMARK; ${ }^{3}$ Sport Individual \& Society, Department of Nutrition Exercise and Sports, University of Copenhagen, Copenhagen, DENMARK; ${ }^{4}$ Research in Childhood Health (RICH), Exercise Epidemiology, Department of Sports Science and Clinical Biomechanics, University of Southern Denmark, Odense, DENMARK; ${ }^{5}$ The Centre of Inflammation and Metabolism (CIM) and The Center for Physical Activity Research, (CFAS), Rigshospitalet, University of Copenhagen, Copenhagen, DENMARK; ${ }^{6}$ The Danish Diabetes Academy, Odense University Hospital, Odense, DENMARK; and ${ }^{7}$ Forest and Landscape College, Department of Geosciences and Natural Resource Management, University of Copenhagen, Fredensborg, DENMARK

\begin{abstract}
SCHNELLER, M. B., P. BENTSEN, G. NIELSEN, J. C. BRØND, M. RIED-LARSEN, E. MYGIND, and J. SCHIPPERIJN. Measuring Children's Physical Activity: Compliance Using Skin-Taped Accelerometers. Med. Sci. Sports Exerc., Vol. 49, No. 6, pp. 1261-1269, 2017. Introduction: Accelerometer-based physical activity monitoring has become the method of choice in many large-scale physical activity (PA) studies. However, there is an ongoing debate regarding the placement of the device, the determination of device wear time, and how to solve a lack of participant compliance. The aim of this study was to assess the compliance of Axivity AX3 accelerometers taped directly to the skin of 9- to 13-yr-old children. Methods: Children in 46 school classes (53.4\% girls, age $11.0 \pm 1.0 \mathrm{yr}$, BMI $17.7 \pm 2.8 \mathrm{~kg} \cdot \mathrm{m}^{-1}$ ) across Denmark wore two Axivity AX3 accelerometers, one taped on the thigh $(n=903)$ and one on the lower back $(n=856)$, for up to 10 consecutive days. Participants were instructed not to reattach an accelerometer should it fall off. Simple and multiple linear regressions were used to determine associations between accelerometer wear time and age, sex, BMI percentiles, and PA level. Results: More than $65 \%$ had $>7 \mathrm{~d}$ of uninterrupted, 24-h wear time for the thigh location and $59.5 \%$ for the lower back location. From multiple linear regressions, PA levels showed the strongest association with lower wear time (thigh: $\beta=-0.231, R^{2}=0.066$; lower back: $\beta=-0.454, R^{2}=0.126$ ). In addition, being a boy, being older (only for lower back), and having higher BMI percentile were associated with lower wear time. Conclusion: Using skin-taped Axivity accelerometers, we obtained $7 \mathrm{~d}$ of uninterrupted accelerometer data with 24-h wear time per day with a compliance rate of more than $65 \%$. Thigh placement resulted in higher compliance than lower back placement. Achieving days with 24-h wear time reduces the need for arbitrary decisions regarding wear time validation and most likely improves the validity of daily life PA measurements. Key Words: ACCELEROMETRY, PA BEHAVIOR, VALIDITY, WEAR TIME
\end{abstract}

\begin{abstract}
Address for correspondence: Mikkel Bo Schneller, M.Sc., Steno Diabetes Center Copenhagen, the Capital Region of Denmark Niels Steensens Vej 6, DK-2820 Gentofte, Denmark; E-mail: mikkel.bo.schneller@regionh.dk.

Submitted for publication October 2016.

Accepted for publication January 2017.

0195-9131/17/4906-1261/0

MEDICINE \& SCIENCE IN SPORTS \& EXERCISE ${ }_{\circledast}$

Copyright $\odot 2017$ The Author(s). Published by Wolters Kluwer Health, Inc. on behalf of the American College of Sports Medicine. This is an openaccess article distributed under the terms of the Creative Commons Attribution-Non Commercial-No Derivatives License 4.0 (CCBY-NC$\mathrm{ND}$ ), where it is permissible to download and share the work provided it is properly cited. The work cannot be changed in any way or used commercially without permission from the journal.
\end{abstract}

DOI: 10.1249 /MSS.0000000000001222
A reliable and valid measure of children's free-living physical activity (PA) applicable in large-scale studes is crucial when linking PA behavior to health outcomes, or when assessing the PA levels of a population or the effects of interventions aiming at increasing daily PA. As it is generally accepted that accelerometers are able to measure PA accurately for multiple days and provide information on PA intensity at any given time, they are widely used to quantify children's free-living PA level and pattern (5). However, the reliability of the measurement is affected by both the attachment method and placement of the accelerometer(s) (20). To reflect real-life behavior and to diminish the effects of day-to-day variation, high-quality measurements require high wear compliance, in terms of many hours of wear time per day and a sufficient number of included days $(1,11)$. Despite the importance of high compliance 
rates, wear time per day usually remains below $24 \mathrm{~h}$ in studies measuring PA in children. This leads to arbitrary decisions regarding wear time validation criteria and inclusion criteria for a valid day, which can significantly affect the compliance rates (12), wear time (7), time spent in different PA intensities (13), and differential bias by exclusion of participants with certain characteristics (27). Furthermore, a review by Cain et al. (5) highlighted the great diversity of methods and criteria used to process accelerometer-based data in studies investigating PA in children published between 2005 and 2010, which makes comparisons between studies difficult.

It is likely that compliance to the accelerometer method is affected by the accelerometer's size and shape, attachment method, attachment site, and/or instructions for use. From a compliance perspective, it may thus be preferable that the accelerometer model is small, lightweight as possible, as well as feasible to use during all daily life conditions for several days. To our knowledge, the Axivity AX3 (Axivity, Newcastle, UK) is currently the smallest $(23 \times 32.5 \times$ $7.6 \mathrm{~mm}, 11 \mathrm{~g}$ ) commercially available research-grade accelerometer-based PA monitor with the ability to sample raw accelerometry data.

Traditionally, accelerometers have been worn at the waist attached to a belt $(28,32,34)$, as this position close to the center of the body mass has been found to offer a strong association between measured accelerations and energy expenditure. Unfortunately, compliance rates for wearing waistmounted accelerometers for multiple days have been relatively low (28). Several recent studies have used an accelerometer placed in a wristband to increase compliance rates $(12,29)$. However, Tudor-Locke et al. (31) recently found that a 24-h wear protocol resulted in higher wear compliance with a waist-mounted accelerometer than in previous studies, including those using a wrist-mounted accelerometer, whereas Howie et al. (14) found no difference in compliance between two identical accelerometers worn concurrently at the hip and the wrist.

In addition to considering validity problems related to wear compliance, it is important to choose an accelerometer placement that provide a valid measure of the PA construct in question. In the current literature, wrist placement has generally been found to be less accurate than waist placement in PA behavior classification $(9,10,17,23,24,30,33,35)$, although certain activities, such as basketball and dancing, are more accurately recorded by wrist-worn accelerometers than hipworn ones (9,30). Thigh-placed accelerometers accurately classify time spent lying down, sitting, standing, and moving for use in sedentary behavior analyses $(18,22)$ and PA types, including cycling and walking up and down stairs (26).

One possible solution to several of the above-mentioned problems associated with accelerometers involves taping waterproof accelerometers directly to the skin of study participants at the waist and the thigh. The aim of this study was to assess the compliance when Axivity AX3 accelerometers were taped directly to the skin of 9- to 13-yr-old children.
More specifically, we will describe wear time patterns and investigate predictors of compliance when accelerometers are taped to the lower back and the thigh.

\section{METHODS}

Setting and study design. The study population was drawn from the TEACHOUT study, a quasi-experimental intervention study of 46 Danish primary school classes. Data collection was performed during the school year August 2014 to June 2015. PA measurements took place between November 2014 and June 2015, after a pilot study was conducted in spring 2014 to test different accelerometer taping methods.

Pilot study of accelerometer taping methods. We conducted a pilot study from March to May 2014 in four classes ( $n=96$ children) from grade four through six using Axivity AX3 accelerometers, testing different methods to find a suitable solution to attaching the accelerometers to the participants' skin with tape. The tested methods included skin cleaning and different types of tape with distinct functions, acting as a bottom and a top layer. The bottom layer was intended to protect the skin, to fixate the accelerometer, and to add strength to the attachment. The top layer was intended to provide strong fixation and be skin-friendly and as waterproof and airtight as possible, but also allow the skin to breathe, keep bacteria out, and be transparent to reveal potential skin reactions. Different types of medical tape cut into various shapes and sizes were tested during the pilot testing. The final tape solution is described in the Data Collection Procedure section.

Sampling and participants. A total of 903 children from 46 third- to sixth-grade classes across 18 schools had an accelerometer attached to their thigh, and 856 of them also had an accelerometer attached to their lower back. The 47 children who only had an accelerometer attached to their thigh did not wear one on their lower back due to a shortage of available accelerometers on some setup days. Table 1 shows participant characteristics.

Accelerometer model. The Axivity AX3 (Axivity) is a three-axis accelerometer measuring $23 \times 32.5 \times 7.6 \mathrm{~mm}$ and weighing $11 \mathrm{~g}$. It is dustproof and waterproof to $1.5 \mathrm{~m}$ and has an operating temperature range of $0{ }^{\circ} \mathrm{C}-65^{\circ} \mathrm{C}$. It includes a real-time clock, $512 \mathrm{MB}$ of built-in memory, $14 \mathrm{~d}$

TABLE 1. Participant characteristics.

\begin{tabular}{|c|c|c|}
\hline Placement & Thigh & Lower Back \\
\hline Total participants & 903 & 856 \\
\hline Girls, $n$ & $478(52.9 \%)$ & $452(52.8 \%)$ \\
\hline Age, yr & $11.0 \pm 1.0(n=787)$ & $11.0 \pm 1.0(n=741)$ \\
\hline Height, cm & $148.1 \pm 8.7(n=897)$ & $148.2 \pm 8.7(n=850)$ \\
\hline Weight, kg & $39.2 \pm 8.6(n=896)$ & $39.4 \pm 8.7(n=849)$ \\
\hline $\mathrm{BMI}, \mathrm{kg} \cdot \mathrm{m}^{-2}$ & $17.8 \pm 2.8(n=895)$ & $17.8 \pm 2.8(n=848)$ \\
\hline BMI percentile ${ }^{a}$ & $49.1 \pm 28.6(n=783)$ & $49.0 \pm 28.5(n=738)$ \\
\hline$\%$ overweight or obese ${ }^{a}$ & 14.3 (112 of 783$)$ & 14.5 (107 of 738$)$ \\
\hline$\%$ obese $^{a}$ & $3.3(26$ of 783$)$ & 3.5 (26 of 738$)$ \\
\hline
\end{tabular}

Age, height, weight, BMI, and BMI percentile are presented as mean \pm SD. ${ }^{a}$ Classification based on Barlow and Dietz (3). 
of recording time at $100 \mathrm{~Hz}$, a temperature and light sensor, and a flexible setup allowing logging frequencies of 12.5$3200 \mathrm{~Hz}$ and a bandwidth range of $2 \mathrm{G}, 4 \mathrm{G}, 8 \mathrm{G}$, or $16 \mathrm{G}$. The accelerometers were initialized to measure at $50 \mathrm{~Hz}$ with $\pm 8 \mathrm{G}$ bandwidth using OmGui version 1.0.0.30 (Newcastle University, UK).

Data collection procedure. Each participant wore two Axivity AX3 accelerometers, which a researcher had attached directly to the skin of their lower back and the front of their thigh using tape (Fig. 1). The participants were instructed to wear the accelerometers at all times (including water activities and sleep) for up to 10 consecutive days and not to reattach an accelerometer if it fell off before the 10-d period ended. The accelerometer on the lower back was placed on the right side, above the upper point of the posterior iliac crest and next to the spine with its positive $x$-axis pointing downward and its negative $z$-axis pointing forward. The accelerometer on the thigh was placed on the medial front of the right thigh, midway between the hip and the knee joints, with its positive $x$-axis pointing downward and its negative $z$-axis pointing forward. Both accelerometers were tape mounted using a four-step protocol. Figure 1 shows the placement of the accelerometers (Fig. 1E) and the four-step protocol (Fig. 1A-D). First, the skin was cleaned using an alcohol wipe. Second, a $30 \times 50-\mathrm{mm}$ piece of Fixomull tape (BSN Medical) with a $10 \times 20-\mathrm{mm}$ piece of double-sided adhesive tape (3 M, Hair-set) on top of it was placed on the clean, dry skin. Third, the accelerometer was placed on the double-sided tape. Fourth, an $80 \times 100-\mathrm{mm}$ piece of Opsite Flexifix (Smith \& Nephew) with rounded corners was placed on top of the accelerometer. Date of birth, height (Leicester Height Measure), and weight (Omron Body Composition) were collected on the day the participant had the accelerometers attached. Height and weight were used to calculate BMI and BMI percentile, using the formula by Barlow and Dietz (3). In addition, each participant was asked to complete a diary during the monitoring period and report why a monitor was no longer worn, if this happened.
Data processing. The $50-\mathrm{Hz}$ raw acceleration data were stored in the original cwa Axivity file format, and also converted into a binary gt $3 \mathrm{x}$ compatible file format using a custom-made add-on to OmGui, to access intensity estimates using the ActiLife (version v6.11.9; ActiGraph, Pensacola, FL) software (15). The data in the gt3x files were stored using a $30-\mathrm{Hz}$ sampling frequency and thus resampled from the original $50 \mathrm{~Hz}$ to avoid the potential bias in the intensity estimations with the ActiLife software (4).

The accelerometer wear time period was determined manually using OmGui based on raw accelerometry and temperature data output for each file as the length of the time interval from the first determined time of wear until the first nonwear time. Times were written into an Excel data sheet, and wear time for each file was calculated. This time period was used as measure of a participant's compliance in wearing the device. Figure 2 shows an example of raw accelerometer data from the $x$-axis and temperature output from a data file for a thigh-mounted accelerometer with markings showing manually determined start and stop time. A full output file was expected to have a size of $260 \mathrm{MB}$ or larger. Devices for which a data file could not be downloaded, or for which the downloaded files had a size of less than $75 \%$ of the expected size, were flagged and investigated manually to determine why this was the case. A data file indicating a technical error as the cause of the loss of actual wear time was classified as a "malfunctioning device." For files that were smaller than expected, if an accelerometer was still attached at the last recorded data point in the data file, and the data file was stopped earlier than $10 \mathrm{~d}$ after attachment, it was identified as a file with "loss of wear time." Files downloaded from malfunctioning devices were included in the analyses.

Subject Log Diaries in ActiLife format were created, which included filename-specific information on start and stop in wear time in two ways for both thigh and lower back placement. First, all participants with nonstop wear time from midnight on the day of monitor attachment and seven
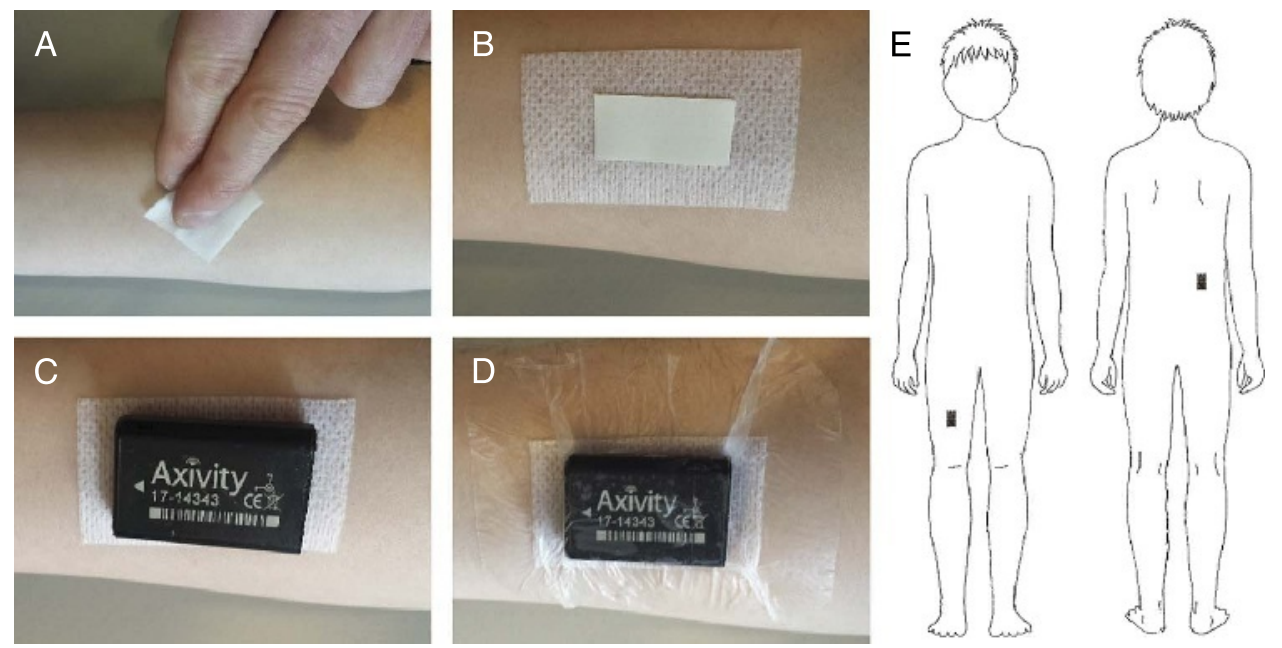

FIGURE 1-Accelerometer tape fixation protocol and placement. 

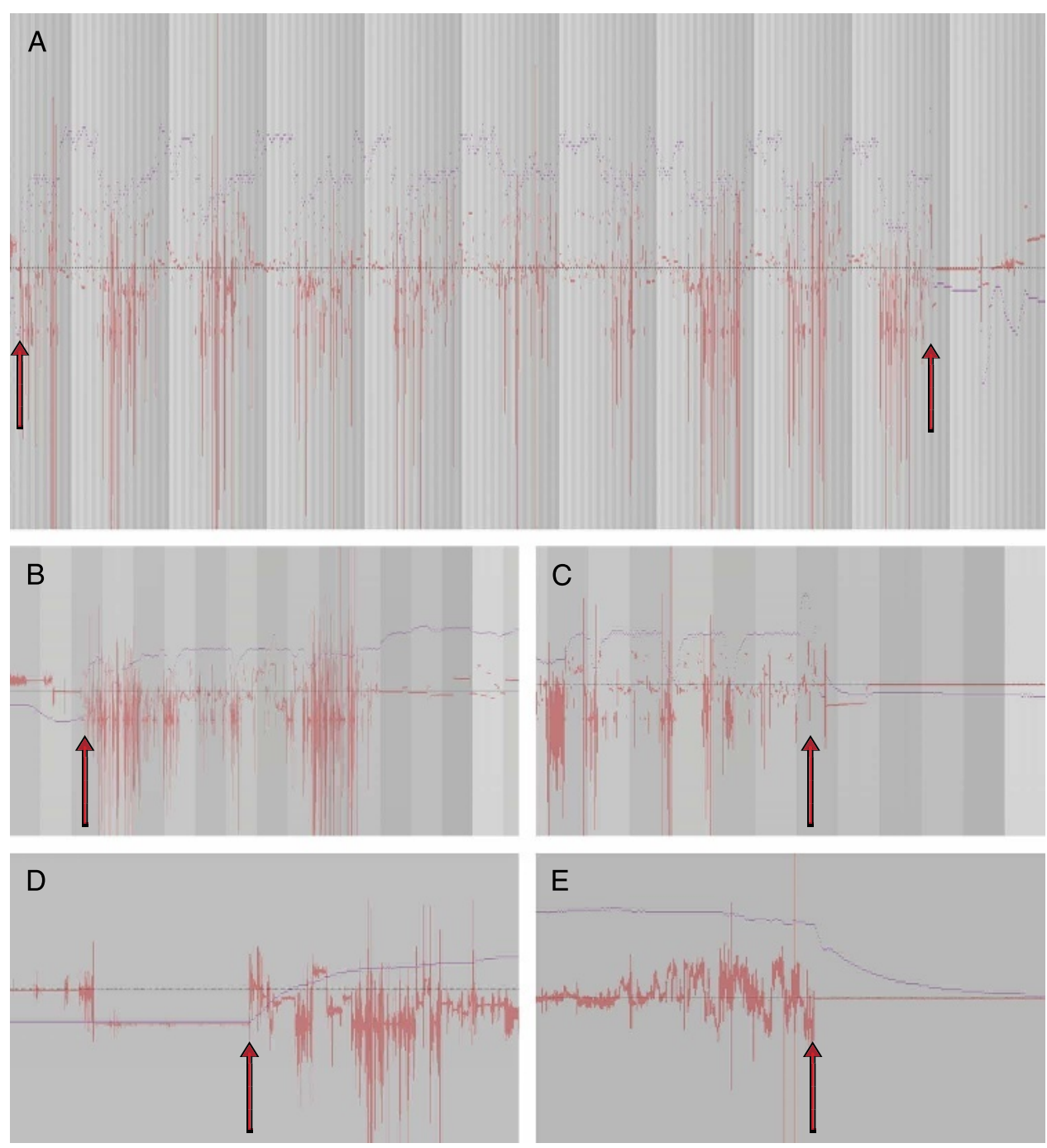

FIGURE 2-Wear time validation example. In all pictures, the dashed line represents $0 g$; the red curves are raw accelerometer $x$-axis data; the purple line represents temperature; and each vertical stripe represents $1 \mathrm{~h}$. A, Ten days of accelerometer measurements. The left red arrow points at the determined start in wear time, and the right red arrow points toward the determined stop in wear time. B, Seventeen hours of accelerometer measurements with the red arrow pointing toward the determined start in wear time. C, Fifteen minutes of accelerometer measurements with the red arrow pointing toward the determined start in wear time. $\mathrm{D}$, Seventeen hours of accelerometer measurements with the red arrow pointing toward the determined stop in wear time. E, Fifteen minutes of accelerometer measurements with the red arrow pointing toward the determined stop in wear time.

full days forward were included in a subject log diary file with dates and times of these $7 \mathrm{~d}$. The log diary information was then applied to the corresponding files in ActiLife. Hourly calculations of vector magnitude for all three axes in counts per minute (VM3) were conducted for each file, and data were exported to Excel. Each participant included (472 at lower back and 534 at thigh) had $168(24 \mathrm{~h} \times 7 \mathrm{~d})$ hourly values of VM3 linked to a specific hour on a specific day. Second, all participants with any wear time had all wear time written into a subject log diary file, which was applied to the corresponding files in ActiLife. One calculated mean value of VM3 for all wear time of each participant was then exported to Excel and linked to the participant.

Statistical analysis. Wear time means \pm SD were calculated for both thigh and lower back placement for all participants who had accelerometers mounted. Accelerometers that were not returned were set to $0.00 \mathrm{~d}$ of wear time and were included in the analyses. Paired $t$-tests were used to check for differences between lower back and thigh wear times for children (and separately for girls and boys) who initially had accelerometers attached on both locations.

One-day intraclass correlation (ICC) values were calculated for each of the $7 \mathrm{~d}$ of the week. Hourly mean VM3 counts per minute values were used to construct whole day ICC. The average ICC values for Monday to Friday (i.e., week day) as well as Saturday and Sunday (i.e., weekend day) were then applied in the Spearman-Brown prophecy formula. We calculated the number of monitoring days necessary to assess day-to-day variability in overall PA with an $80 \%$ reliability using only participants with data from seven full days of device wear time for thigh and lower back placements separately. 
TABLE 2. Participant wear time, device performance, and reasons for nonwear

\begin{tabular}{lcc}
\hline Placement & Thigh & Lower Back \\
\hline Total participants & 903 & 856 \\
Mean days of wear time & $7.29 \pm 3.27$ & $6.80 \pm 3.48$ \\
Days of monitoring required to achieve $80 \%$ reliability in VM3CPM & output on... \\
Unspecified days & 4.2 & 3.1 \\
Week days/weekend days & $5.2 / 2.5$ & $3.5 / 2.3$ \\
Accelerometers not returned & $37(4.1 \%)$ & $35(4.1 \%)$ \\
Nondownloadable files & $8(0.9 \%)$ & $3(0.4 \%)$ \\
Files $<75 \%$ of expected size & ( & $27.3 \%)$ \\
Files with lost data due to device malfunction & $22(2.4 \%)$ & $15(1.8 \%)$ \\
$\quad$ Mean days included per file with lost data ${ }^{b}$ & $4.04 \pm 2.36$ & $2.83 \pm 1.84$ \\
Participants with data file & $858(95.0 \%)$ & $818(95.6 \%)$ \\
Reason for stop in wear time & & \\
Participants with diary and $<8$ d of wear time & $202(65.4 \%)^{c}$ & $232(66.5 \%)^{c}$ \\
Deliberately removed & $32(15.8 \%)$ & $47(20.3 \%)$ \\
$\quad$ Mean days of wear time before removed & $4.43 \pm 2.01$ & $3.60 \pm 1.87$ \\
Fell off & $68(33.7 \%)$ & $74(31.9 \%)$ \\
$\quad$ Mean days of wear time before falling off & $4.79 \pm 2.05$ & $4.61 \pm 2.10$ \\
\hline
\end{tabular}

Values are reported as mean $\pm \mathrm{SD}, n$, or $n(\%)$.

${ }^{a}$ Expected full file size was minimum $260 \mathrm{MB}$; files were flagged when $<75 \%$ of expected size.

${ }^{b}$ Average number of days of wear time per file included in the analysis before a technical error caused some extent of lost data, as the accelerometer was still worn and the data file ended prematurely.

${ }^{c}$ Number of participants with $<8 \mathrm{~d}$ of continuous accelerometer wear time were 309 for thigh and 349 for lower back.

Linear regressions were used to determine associations between age, sex, BMI percentile, and VM3 as independent variables and accelerometer wear time on the thigh and lower back, respectively, as dependent variables on the other side. VM3 in counts per minute was calculated as one mean value for the full length of each participant's wear time and used as indicator of the effect of overall PA on compliance. Unadjusted linear regressions and multiple linear regressions were used to determine the independent associations between each predictor variable and accelerometer wear time for the thigh and lower back, respectively.

The proportions of participants wearing accelerometers for each number of consecutive 24-h wear time days (a valid day) and their $95 \%$ confidence intervals were calculated by placement site for all participants who started out wearing accelerometer(s).

Analyses were performed using Stata 14.2 (StataCorp, College Station, TX), and the significance level was set to $P<0.05$.

Research ethics. All parents provided informed consent, and the study was approved by the Danish Data Authorities (ref. no. 2014-54-0638). The Scientific Ethical Committee in the Capital Region of Denmark determined that the project did not require formal ethical approval, as it did not involve biomedical measures (protocol number H-4-2014-FSP).

\section{RESULTS}

Table 2 shows participant wear time, device performance, and reasons for nonwear. Mean wear time was $7.29 \pm 3.27 \mathrm{~d}$ for thigh and $6.80 \pm 3.48 \mathrm{~d}$ for lower back, resulting in higher thigh compared with lower back wear time $(95 \%$ confidence interval $[\mathrm{CI}]=0.18$ to $0.81, P=0.001)$. Mean thigh wear time was $0.66 \mathrm{~d}$ higher for boys $(95 \% \mathrm{CI}=0.18$ to $1.14, P=0.003)$ and $0.36 \mathrm{~d}$ higher for girls $(95 \% \mathrm{CI}=$ -0.35 to $0.75, P=0.037$ ) compared with lower back wear time for the corresponding sex. To achieve $80 \%$ reliability, a minimum of $3.1 \mathrm{~d}$ of measurement was needed at the lower back and $4.2 \mathrm{~d}$ of measurement at the thigh; this was achieved by $82.2 \%$ and $82.7 \%$ of participants, respectively. More week days than weekend days where required for both placements (i.e., thigh, 5.2 vs 2.5; lower back, 3.5 vs 2.3), and more week days were required at the thigh compared with the lower back to achieve $80 \%$ reliability of monitored VM3CPM for an individual.

Reasons for no longer wearing accelerometers were provided by 100 participants for the thigh-placed monitor and 121 for lower back in cases with $<8 \mathrm{~d}$ of wear time. Of these cases, $32 \%$ and $38 \%$ of monitors were deliberately removed from the thigh and lower back, respectively, whereas $68 \%$ and $62 \%$ fell off because of the lack of adherence of the tape at the thigh and lower back, respectively. Mean wear time in days before deliberately removing a monitor was $0.83 \mathrm{~d}$ higher at the thigh than at the lower back placement.

Table 3 shows the proportion of participants with continuous 24-h accelerometer wear time by number of days from accelerometer attachment for thigh and lower back. The proportion of participants who had $\geq 1 \mathrm{~d}$ of continuous 24-h compliance was $92.5 \%$ for thigh-mounted and $91.7 \%$ for lower back-mounted accelerometers. For $\geq 7 \mathrm{~d}$, this figure was $65.7 \%$ for thigh-mounted and $59.5 \%$ for lower backmounted accelerometers. The proportion of participants with any given number of minimum days of continuous 24-h compliance was higher for thigh- compared with lower backmounted accelerometers by an average of $5.0 \%$.

Table 4 shows the associations from unadjusted linear regressions and multiple linear regressions between accelerometer wear time and sex, weight status, age, and VM3. VM3 showed the strongest associations with variation in wear time explaining $8.1 \%$ (thigh) and $14.7 \%$ (lower back) when unadjusted for other predictors and $6.6 \%$ (thigh) and $12.6 \%$ (lower back) when tested independent of other predictors. Sex showed the second strongest association in unadjusted linear regression explaining 6.0\% (thigh) and $7.2 \%$ (lower back), but when tested in multiple linear

TABLE 3. Percentage of participants by days of $24-\mathrm{h}$ wear time by accelerometer placement site.

\begin{tabular}{lcc}
\hline Days & Thigh, $\boldsymbol{n}=\mathbf{9 0 3}$ & Lower Back, $\boldsymbol{n}=\mathbf{8 5 6}$ \\
\hline$\geq 1$ & $92.5(90.7-94.2)$ & $91.7(89.9-93.5)$ \\
$\geq 2$ & $89.5(87.5-91.5)$ & $86.4(84.2-88.7)$ \\
$\geq 3$ & $84.6(82.3-87.0)$ & $79.4(76.8-82.1)$ \\
$\geq 4$ & $79.4(76.8-82.0)$ & $73.1(70.2-76.0)$ \\
$\geq 5$ & $74.8(71.9-77.6)$ & $67.9(64.8-70.9)$ \\
$\geq 6$ & $71.0(68.0-73.9)$ & $65.0(61.8-68.1)$ \\
$\geq 7$ & $65.7(62.6-68.8)$ & $59.5(56.3-62.7)$ \\
$\geq 8$ & $60.4(57.2-63.5)$ & $55.1(51.9-58.4)$ \\
$\geq 9$ & $51.5(48.2-54.8)$ & $45.9(42.7-49.2)$ \\
\hline
\end{tabular}

Values show compliance rate in percent $(95 \% \mathrm{Cl})$ for all participants. The reported numbers are based on the entire sample of children who had an accelerometer placed on the specific site and include devices not returned and malfunctioning devices. 


\begin{tabular}{|c|c|c|c|c|c|c|c|c|c|c|c|c|}
\hline & \multicolumn{6}{|c|}{ Thigh Wear Time } & \multicolumn{6}{|c|}{ Lower Back Wear Time } \\
\hline & $n$ & $\boldsymbol{\beta}$ & \multicolumn{2}{|c|}{$95 \% \mathrm{Cl}$} & $R^{2}$ & $P$ & $n$ & $\beta$ & \multicolumn{2}{|c|}{$95 \% \mathrm{Cl}$} & $R^{2}$ & $P$ \\
\hline \multicolumn{13}{|c|}{ Linear regression, unadjusted } \\
\hline Boys vs girls & 856 & 1.410 & 1.034 & 1.785 & 0.060 & $<0.001$ & 817 & 1.731 & 1.303 & 2.159 & 0.072 & $<0.001$ \\
\hline BMI percentile* & 740 & -0.021 & -0.028 & -0.014 & 0.043 & $<0.001$ & 701 & -0.020 & -0.028 & -0.011 & 0.031 & $<0.001$ \\
\hline $\mathrm{Age}^{*}$ & 743 & -0.047 & -0.253 & 0.160 & 0.000 & 0.657 & 704 & -0.336 & -0.571 & -0.101 & 0.011 & 0.005 \\
\hline VM3* & 855 & -0.253 & -0.310 & -0.195 & 0.081 & $<0.001$ & 810 & -0.469 & -0.546 & -0.391 & 0.148 & $<0.001$ \\
\hline \multicolumn{13}{|c|}{ Multiple linear regression } \\
\hline Boys vs girls & 737 & 0.802 & 0.407 & 10.198 & 0.021 & $<0.001$ & 693 & 0.575 & 0.120 & 10.031 & 0.009 & 0.013 \\
\hline BMI percentile* & 737 & -0.021 & -0.028 & -0.014 & 0.049 & $<0.001$ & 693 & -0.018 & -0.026 & -0.011 & 0.033 & $<0.001$ \\
\hline $\mathrm{Age}^{*}$ & 737 & -0.168 & -0.365 & 0.029 & 0.004 & 0.095 & 693 & -0.571 & -0.794 & -0.348 & 0.036 & $<0.001$ \\
\hline VM3* & 737 & -0.231 & -0.294 & -0.168 & 0.066 & $<0.001$ & 693 & -0.454 & -0.544 & -0.365 & 0.126 & $<0.001$ \\
\hline
\end{tabular}

$P<0.05$ are in bold; $n$ equals sample size included in statistical test.

${ }^{\star}$ Difference with an increase of one in BMI percentile, $1 \mathrm{yr}$ in age and 100 counts per minute in VM3.

regression, the explanation of variation in wear time decreased to $2.1 \%$ (thigh) and $0.9 \%$ (lower back).

\section{DISCUSSION}

We evaluated compliance in a study measuring children's free-living PA for 10 consecutive days using two tape-mounted Axivity AX3 accelerometers. With seven valid days of 24-h wear time per day as the inclusion criterion, we obtained a compliance of $65.7 \%$ for thigh placement and $59.5 \%$ for lower back placement. Using a full week of accelerometerderived PA measurements as inclusion criterion in studies investigating daily life PA may increase the validity of the measurements by removing subjective decisions in defining nonwear time. In addition, using a full week will eliminate the need to take into consideration whether weekly occurring types of days are included proportionally representatively of daily life. Weekly occurring types of days for children could be school days with PE, school days without PE (or other potential weekly occurring educational practices), and weekend days. In addition to these cyclical recurring activities, children's PA and sedentary behavior include substantial day-to-day variation. Fairclough et al. (11) investigated the reproducibility of objectively measured daily MVPA in children (7-11 yr) using waist-mounted ActiGraph monitors. They found that because of day-to-day variability, to achieve $80 \%$ reliability, eight valid measurement days ( $\geq 10.1 \mathrm{~h}$ of wear time) for boys and $10 \mathrm{~d}$ for girls were needed. Our data (Table 2) show a need to include between 2.3 and 5.2 valid days with $24 \mathrm{~h}$ of monitoring to achieve $80 \%$ reliability of overall PA level (VM3CPM), depending on bodily placement and day of week. Participants in our study achieved at least $80 \%$ reliability in more than $82 \%$ of cases for both monitor placements.

Recent accelerometry-based free-living PA measurements in larger samples of children have shown compliance rates for waist-mounted accelerometers of 73.5\% (76.7\% after requesting rewear) in the US ISCOLE sample $(n=491$, mean age $=9.9 \mathrm{yr})(31), 66.7 \%$ in the European multinational ENERGY $(n=1082$, mean age $=11.7 \mathrm{yr})$ sample (11), and 64.3\% in the NHANES 2003-2004 $(n=586$, mean age $=10.4 \mathrm{yr})$ cycle $(28)$ when a period of $4 \mathrm{~d}$ with $\geq 10-\mathrm{h}$ wear time per day was considered to denote a valid participant. In our study, we found a compliance of $79.4 \%$ for thigh and $73.1 \%$ for lower back with at least $4 \mathrm{~d}$ of $24-\mathrm{h}$ wear time. In comparison, we achieved at least similar compliances rates, but with 24-h wear time per day and avoiding any arbitrary decisions regarding wear time validation procedures and related validity problems. The thighplaced accelerometer had a higher wear time compliance compared with the waist-placed one; this is promising with regard to the fact that the thigh-placed accelerometer has a well-validated ability to reflect PA behavior through activity type recognition (26), particularly sedentary behavior $(16,18$, $22,24)$. ActivPAL monitors have been extensively used taped to the thigh in adult studies accompanied by compliance rates. A recent review summarized compliance rates from four studies where participants concurrently wore monitors on the thigh (ActivPAL) and hip (ActiGraph) (8). The proportion of participants with seven or more valid days ( $>10 \mathrm{~h}$ of wear time) ranged between $44.9 \%$ and $90.5 \%$ and mean wear during waking hours between 15.3 and $15.8 \mathrm{~h} \cdot \mathrm{d}^{-1}$ for thigh monitors, whereas these numbers where $66.9 \%-79.3 \%$ and $14.3-15.6 \mathrm{~h} \cdot \mathrm{d}^{-1}$ for hip monitors. To the best of our knowledge, no studies have reported compliance rates of ActivPAL taped to the thigh of children.

In addition, the tape-mounted solution diminishes or removes the influence of a range of shortcomings normally associated with population-based PA monitoring, such as intentional nonwearing time and changing the monitor's position on the body (20). Taking into account the 24-h wear time per day and compliance rates, the tape-mounting method used in this study improves data quality compared with previous accelerometry methods.

Possible explanations of the higher wear time compliance rates for a thigh-placed accelerometer compared with a lower back-placed one in the children in this study could be either the children's perceived acceptability being higher at the thigh compared with the lower back, the tape solution working better at the thigh, or a combination of the two. Also, our results showed a larger proportion of participants deliberately removing monitors, and doing it earlier, from the lower back compared with the thigh. Through participant feedback from our pilot study, main study, and diary information, we believe that these differences in occurrence rate and wear time can mainly be attributed to a higher frequency of skin irritation. 
We suspect that greater mechanical movement and higher skin temperatures, leading to more sweat production, at the lower back compared with thigh might be reasons for this difference. This suspicion may be further strengthened by the negative correlation between overall PA level and wear time (VM3 in Table 4) being stronger for lower back compared with thigh. A study by Fairclough et al. (12) interpreted higher compliance rates for a wrist-placed accelerometer compared with a waistplaced one among children as higher perceived acceptability and preference for wrist-worn devices over waist-worn ones when tested concurrently, although they used different accelerometer models for the two placements. The higher removal rates and the lower wear time for skin-taped accelerometers at the lower back compared with thigh found in this study suggest that the perceived acceptability was higher for the thigh. Our results show that being less physically active, a girl, younger, and having a lower BMI percentile are indicators of a higher wear time for lower back accelerometers. Higher BMI percentiles are related to greater subcutaneous adiposity, which may result in more mechanical movement and heat production (and sweat) when active. For thigh-mounted accelerometers, we found the same indicators except for age. Findings on sex differences in compliance from other studies are mixed. Compliance for girls was also found to be higher in the AHEAD study (2), whereas no differences were found between the sexes in either the ISCOLE (31) or the NHANES 2003-06 (28) studies.

The taping method, and our accelerometer attachment protocol in general, may also have affected the economic and practical aspects of the data collection, as we lost 72 of the 1759 accelerometers (4.1\%) distributed to the participants. The international survey study ISCOLE conducted measurements in a similar age-group in 12 countries, totaling 7314 distributed accelerometers, and only lost 29 of them $(0.4 \%)$ (32). The higher prevalence of lost accelerometers in our study compared with ISCOLE might have been caused by the use of different accelerometer models and attachment methods, differences in the sample population, compliance enhancing strategies, and instructions for wear. We used the black Axivity AX3, whereas ISCOLE used the red ActiGraph GT3X+, and the AX3 is approximately a quarter of the size of the GT3X+. The color difference and smaller size may have made it easier to misplace the AX3 compared with the GT3X+. The tape placement in combination with the instruction to wear the accelerometer for $10 \mathrm{~d}$ or until it fell off may have created cases in which an AX3 fell off without the participant knowing when and where this had happened, but in most cases, the participants did not report to us why they had lost it. With the belt fixation used in ISCOLE, it seems less likely that accelerometers would be lost without participants realizing it. ISCOLE used phone calls, daily visits to the school, and small daily incentives like erasers and stickers in their complianceenhancing strategy. We asked teachers to remind the children to return their accelerometers the day before we collected them at the school, but nothing more. It is not known whether these factors were related to the higher number of lost devices, or to what degree, but it seems likely that they all contributed. However, we believe that $24 \mathrm{~h}$ of valid measurements per day and as little contact with the participants as possible, as part of the overall data collection strategy to affect their behavior minimally, is more important than a higher rate of lost devices.

Strengths and limitations. To our knowledge, this is the first study to directly compare wear compliance involving two accelerometer placements on children, using the same accelerometer model and attachment method in the same sample, worn concurrently to isolate the placement as a variable for compliance. It is also, to our knowledge, the first study to successfully collect 24 -h consecutive PA measurements for several days in children with no nonwear time breaks using accelerometry. We believe that the 24-h consecutive PA measurement for several days with no nonwear time is a highly important methodological design feature for a study intending to investigate habitual free-living PA.

In the reliability analysis, we chose VM3 as outcome to make calculations comparable between bodily placements without grouping data on an ordinal scale via cut points, as no well-validated cut points for PA intensities exists for a thighplaced monitor. A strength is the inclusion 472 (lower back) and 534 (thigh) participants, all with $7 \mathrm{~d}$ of 24-h data from Monday to Sunday for both analyses. However, by using the ICC for all (unspecified) days to report days of monitoring required to obtain $80 \%$ reliability of day-to-day variation in PA, we considered each day randomly sampled when presenting the proportion achieving this number. This is a limitation as days were not randomly sampled, which is illustrated by our findings, showing that more days where needed on week days compared with weekend days to achieve the same reliability.

We consciously chose a protocol including up to 10 consecutive days of PA measurements and have reported the part of the sample with a minimum wear time of $n$ days to provide more in-depth information on the performance of the methods used. This makes the study easier to compare to previous studies with different inclusion criteria in number of valid days and at the same time extends to future demands for an increased number of days of measurements to better represent daily life cycles.

We applied clear criteria and used a wear time validation process, with an inclusion of temperature and raw accelerometry data to identify wear time. The inclusion of temperature to identify wear time showed a potential to increase the accuracy both manually and automatically in tape-mounted accelerometer protocols, as temperature output had a highly characteristic inclination slope when the accelerometer was attached and a similar declination slope when it was detached, which can be seen in Figure 2. Manually determining wear time took on average approximately $90 \mathrm{~s}$ per file, which corresponded to $\sim 42 \mathrm{~h}$ when applied to the 1676 data files. An automatic algorithm to detect wear times was trained on the data sets in the current study with similar results as manually determined for both placements. We chose not to use the automatic algorithm because it is yet to 
be validated against a gold standard. However, automatic detection of wear time will be applicable in the near future and thereby eliminate a time consuming step in the data analysis.

The chosen protocol with no reattachments and only one continuous wear period per accelerometer minimized the number of cases in which we could have mistakenly included or excluded invalid data at the start and the end of the wear time period. This was done by instructing participants not to put an accelerometer that had fallen off back on and by providing no extra tape for reattachment purposes. This protocol also made the tape solution an especially critical part of the measurement method and resulted in important challenges that had to be overcome in our data collection protocol. Our taping method required approximately 2 min per participant to attach each accelerometer, and some practice to fit the top tape layer well. In our experience, fitting the top tape layer well included attaching it without touching the edges of the adhesive side of the tape and avoiding folds and air bubbles in the connection to the skin. Unfortunately, it was impossible to avoid this completely when using a flat piece of tape to cover the box-shaped accelerometer.

Because of accelerometers being returned late and lost, we faced challenges in having enough of them in stock on data collection days, especially toward the end of our 8-month data collection period. We spent a considerable amount of time contacting teachers trying to reclaim accelerometers as quickly as possible but still, at its worst, had approximately $60 \%$ of our accelerometers not returned in time or lost. A direct consequence of this was that 47 children only had an accelerometer taped to their thigh rather than to both their thigh and lower back as intended. During our data collection period, we spent approximately $5 \mathrm{~h} \cdot \mathrm{wk}^{-1}$ tracking down missing accelerometers and contacting participants or contact persons at the participating sites. Precautions to lose as few devices as possible should be prioritized when possible to obtain high compliance rates and minimize data, equipment and economic loss.

We would recommend using the current taping protocol as part of larger surveillance protocol, although a more simple taping protocol should be developed before use in postal surveillance studies. If applying this protocol in its current form, researchers should set aside sufficient time for preparing tape, attaching monitors, determining wear times, and retrieving monitors from participants. In addition, using disposable tape rather than a reusable belt or band comes at a price per monitor attached depending on price of tape, and our higher rate of lost devices may also affect the total data collection cost. We spent approximately $40 €$ in tape and $533 €$ in lost Axivity AX3's per 100 accelerometers attached.

A limitation of our analysis was the lack of seasonal/ weather information. We regard this as a limitation because a review has suggested that PA levels of children are negatively affected by precipitation, high wind speeds, and lower temperatures (6), and we found overall PA to be correlated negatively to wear time. Also, it is suggested that the effects of these weather characteristics affect individuals differently according to sex, age, and weight status (21). Seasonal/ weather characteristics may therefore affect wear time, but to which extent is unknown.

Another choice in our protocol was to include lower backand thigh-mounted accelerometers, but not a wrist-mounted one, although wrist-placed accelerometers have been reported to result in higher wear compliance than waist-placed ones in some studies $(12,29)$. We made this decision based on the lower validity of transforming data from a wrist-worn accelerometer obtained in conditions resembling free-living conditions into PA intensities, energy expenditure, and activity type classification compared with a hip-worn one in current data transformation methods $(10,17,23,30)$. However, the literature is not conclusive in this area, and new data processing techniques currently emerge at a rapid pace, which does not seem to slow down any time soon given the recent large surveillance studies like UK Biobank and NHANES using wrist accelerometers. One recent study using an artificial neural network to detect a range of 10 activity categories found higher overall classification accuracy for wrist-placed monitors compared with thigh, and for thigh compared with hip, when tested on adults in semistandardized settings (19). Also, Shiroma et al. (25) found that PA measured using accelerometers worn at the wrist and hip accumulates similarly over time. Therefore, it would have been a strength to include an additional sensor tape mounted on the wrist of the participants in this study.

\section{CONCLUSIONS}

We found the use of a tape-mounted Axivity AX3 to be a method with high compliance for use in large-scale studies to measure PA behavior in children. It is possible to measure 10 consecutive days with 24-h wear time using Axivity AX3 accelerometers attached directly to the skin of 9- to 13-yrold children using tape. Also, the compliance rates we achieved for a thigh-placed monitor were similar to the highest previously reported compliance for wrist placement in children, but at the same time, we had increased the required wear time per day to $24 \mathrm{~h}$. Lastly, the thigh placement was found to be superior, in terms of compliance, to lower back placement. The tape solution should be developed further to improve wear time duration and make the attachment process easier.

This project was funded by TrygFonden and Innovation Fund Denmark. The funders were not involved in the study design, analyses, interpretation or writing, or the decision to submit this article. The authors thank Siri J. Egede for her large contribution in all parts of the data collection and Mads Bølling, Camilla R. Otte, Karen Barfod, and Niels Ejbye-Ernst for their efforts in recruiting participants to the TEACHOUT study. They further thank the participating children, teachers, schools, and municipalities. The results of the study are presented clearly, honestly, and without fabrication, falsification, or inappropriate data manipulation and do not constitute endorsement by the American College of Sports Medicine. The authors declare no conflict of interest. 


\section{REFERENCES}

1. Aadland E, Ylvisaker E. Reliability of objectively measured sedentary time and physical activity in adults. PLoS One. 2015;10(7):e0133296.

2. Audrey S, Bell S, Hughes R, Campbell R. Adolescent perspectives on wearing accelerometers to measure physical activity in populationbased trials. Eur J Public Health. 2013;23(3):475-80.

3. Barlow SE, Dietz WH. Obesity evaluation and treatment: expert committee recommendations. The Maternal and Child Health Bureau, Health Resources and Services Administration and the Department of Health and Human Services. Pediatrics. 1998;102(3):E29.

4. Brønd JC, Arvidsson D. Sampling frequency affects the processing of ActiGraph raw acceleration data to activity counts. $J$ Appl Physiol (1985). 2016;120(3):362-9.

5. Cain KL, Sallis JF, Conway TL, Van Dyck D, Calhoon L. Using accelerometers in youth physical activity studies: a review of methods. J Phys Act Health. 2013;10(3):437-50.

6. Chan CB, Ryan DA. Assessing the effects of weather conditions on physical activity participation using objective measures. Int $J$ Environ Res Public Health. 2009;6(10):2639-54.

7. Colley R, Connor Gorber S, Tremblay MS. Quality control and data reduction procedures for accelerometry-derived measures of physical activity. Health Rep. 2010;21(1):63-9.

8. Edwardson CL, Winkler EAH, Bodicoat DH, et al. Considerations when using the activPAL monitor in field-based research with adult populations. $J$ Sport Health Sci. Available online 3 February 2016, DOI: http://dx.doi.org/10.1016/j.jshs.2016.02.002.

9. Ellis K, Kerr J, Godbole S, Lanckriet G, Wing D, Marshall S. A random forest classifier for the prediction of energy expenditure and type of physical activity from wrist and hip accelerometers. Physiol Meas. 2014;35(11):2191-203.

10. Ellis K, Kerr J, Godbole S, Staudenmayer J, Lanckriet G. Hip and wrist accelerometer algorithms for free-living behavior classification. Med Sci Sports Exerc. 2016;48(5):933-40.

11. Fairclough SJ, Butcher ZH, Stratton G. Whole-day and segmentedday physical activity variability of northwest England school children. Prev Med. 2007;44(5):421-5.

12. Fairclough SJ, Noonan R, Rowlands AV, Van Hees V, Knowles Z, Boddy LM. Wear compliance and activity in children wearing wrist- and hip-mounted accelerometers. Med Sci Sports Exerc. 2016;48(2):245-53.

13. Herrmann SD, Barreira TV, Kang M, Ainsworth BE. Impact of accelerometer wear time on physical activity data: a NHANES semisimulation data approach. Br J Sports Med. 2014;48(3):278-82.

14. Howie EK, McVeigh JA, Straker LM. Comparison of compliance and intervention outcomes between hip- and wrist-worn accelerometers during a randomized crossover trial of an active video games intervention in children. $J$ Phys Act Health. 2016;13(9):964-9.

15. Judge D, Maygarden J. GT3X File Format. San Fransisco (CA): GitHub, Inc.; [cited 2016 May 4]. Available from: https://github. com/actigraph/GT3X-File-Format

16. Kim Y, Barry VW, Kang M. Validation of the ActiGraph GT3X and activPAL accelerometers for the assessment of sedentary behavior. Meas Phys Educ Exerc Sci. 2015;19(3):125-37.

17. Kim Y, Lee JM, Peters BP, Gaesser GA, Welk GJ. Examination of different accelerometer cut-points for assessing sedentary behaviors in children. PLoS One. 2014;9(4):e90630.

18. Lyden K, Kozey Keadle SL, Staudenmayer JW, Freedson PS. Validity of two wearable monitors to estimate breaks from sedentary time. Med Sci Sports Exerc. 2012;44(11):2243-52.
19. Montoye AH, Pivarnik JM, Mudd LM, Biswas S, Pfeiffer KA. Comparison of activity type classification accuracy from accelerometers worn on the hip, wrists, and thigh in young, apparently healthy adults. Meas Phys Educ Exerc Sci. 2016;20(3):173-83.

20. Pedisic Z, Bauman A. Accelerometer-based measures in physical activity surveillance: current practices and issues. Br J Sports Med. 2015;49(4):219-23.

21. Rich C, Griffiths LJ, Dezateux C. Seasonal variation in accelerometer-determined sedentary behaviour and physical activity in children: a review. Int J Behav Nutr Phys Act. 2012; 9:49.

22. Ridgers ND, Salmon J, Ridley K, O'Connell E, Arundell L, Timperio A. Agreement between activPAL and ActiGraph for assessing children's sedentary time. Int $J$ Behav Nutr Phys Act. 2012;9:15.

23. Rosenberger ME, Haskell WL, Albinali F, Mota S, Nawyn J, Intille S. Estimating activity and sedentary behavior from an accelerometer on the hip or wrist. Med Sci Sports Exerc. 2013; 45(5):964-75.

24. Rowlands AV, Olds TS, Hillsdon M, et al. Assessing sedentary behavior with the GENEActiv: introducing the sedentary sphere. Med Sci Sports Exerc. 2014;46(6):1235-47.

25. Shiroma EJ, Schepps MA, Harezlak J, et al. Daily physical activity patterns from hip- and wrist-worn accelerometers. Physiol Meas. 2016;37(10):1852-61.

26. Skotte J, Korshøj M, Kristiansen J, Hanisch C, Holtermann A. Detection of physical activity types using triaxial accelerometers. $J$ Phys Act Health. 2014;11(1):76-84.

27. Toftager M, Kristensen PL, Oliver M, et al. Accelerometer data reduction in adolescents: effects on sample retention and bias. Int $J$ Behav Nutr Phys Act. 2013;10:140.

28. Troiano RP, Berrigan D, Dodd KW, Masse LC, Tilert T, McDowell M. Physical activity in the United States measured by accelerometer. Med Sci Sports Exerc. 2008;40(1):181-8.

29. Troiano RP, McClain JJ, Brychta RJ, Chen KY. Evolution of accelerometer methods for physical activity research. $\mathrm{Br} J$ Sports Med. 2014;48(13):1019-23.

30. Trost SG, Zheng Y, Wong WK. Machine learning for activity recognition: hip versus wrist data. Physiol Meas. 2014;35(11): 2183-9.

31. Tudor-Locke C, Barreira TV, Schuna JM Jr, et al. Improving wear time compliance with a 24-hour waist-worn accelerometer protocol in the International Study of Childhood Obesity, Lifestyle and the Environment (ISCOLE). Int J Behav Nutr Phys Act. 2015;12:11.

32. Tudor-Locke C, Mire EF, Dentro KN, et al. A model for presenting accelerometer paradata in large studies: ISCOLE. Int J Behav Nutr Phys Act. 2015;12:52.

33. Van Hees VT, Golubic R, Ekelund U, Brage S. Impact of study design on development and evaluation of an activity-type classifier. J Appl Physiol (1985). 2013;114(8):1042-51.

34. Yıldırım M, Verloigne M, de Bourdeaudhuij I, et al. Study protocol of physical activity and sedentary behaviour measurement among schoolchildren by accelerometry-cross-sectional survey as part of the ENERGY-project. BMC Public Health. 2011; $11: 182$.

35. Zhang S, Rowlands AV, Murray P, Hurst TL. Physical activity classification using the GENEA wrist-worn accelerometer. Med Sci Sports Exerc. 2012;44(4):742-8. 